\title{
Animation/Simulation for Construction \\ Planning and Scheduling
}

\author{
Stephen Ditlinger, Software Engineer \\ Kermit Gates, Software Engineer \\ Bechtel Power Corporation \\ 12440 East Imperial Highway \\ Norwalk, California 90650
}

\begin{abstract}
Construction CAE is a simulation, planning, and scheduling software system that integrates a Computer Aided Design (CAD) system with a relational database management system (RDBMS) and cost control tools. The system takes the CAD three-dimensional model data as input and stores it in the RDBMS. The data is then extracted from the RDBMS into the system allowing a user to perform construction simulation on all items within the model. A user is able to graphically and interactively assign access paths, manipulate material handling equipment, and define activities for each item in the model. The user may also interact with the system to assign dependencies between the defined activities. The system utilizes the assigned activities and dependencies and critical path method (CPM) scheduling to create an animated construction sequence which may be displayed on the workstation monitor and recorded onto videotape. Initial cost/schedule reports as well as those required for project chronicling are supported through an interface to a Work Breakdown Structure (WBS) and a client's existing schedule reporting system.
\end{abstract}

\section{INTRODUCTION}

With the present business climate of close competition and narrow profit margins, every opportunity to ensure project completion within cost and schedule constraints needs to be exploited. For this reason, project management techniques and tools have been widely accepted and implemented throughtout the $\mathrm{A} / \mathrm{E} / \mathrm{C}$ industry [1]. The functional requirements of general project management are:

Ebjective Setting - Defining goals for a project.

Project Planning - Defining a network of actions and a precedence relation showing their relative order.

Project Scheduling - Linking the plan into a frame of time as well as 
smoothing out resource peaks.

Chronicling and Analysis - Measuring job progress and replanning, rescheduling when conditions change.

Construction management applies these principles of general project management along with the classical network scheduling method of CPM to construction.

CPM is an algorithmic way to accomplish objective setting, project planning and project scheduling. The project plan is a directed acyclic graph that provides a model of the plan's actions and precedences. Project goals are shown as milestones in the plan. There are two different graph representations used in the CPM planning process: precedence diagram method (PDM) and arrow diagram method (ADM). In PDM, actions are represented as nodes in the graph and precedences are represented as edges. In ADM, actions are represented as edges and precedences are represented as nodes.

Chronicling and analysis requires human evaluation (by a planning engineer) of qualitative data and interaction with the project management team. The result of these analyses are corrective actions to ensure timely project completion. Analysis can be very difficult since this requires input, such as material, labor, and equipment availability, from many different sources.

\section{Integrating Tools}

The main goal of the Construction CAE system is to combine existing design and project management tools with an animation system and an RDBMS. By doing so, data assimilation by a planning engineer is simplified since all related data references a CAD model component. For instance: the CAD model provides graphics data for a component; a simulation system provides simulation data; a topdown planning system provides plan and schedule data; a project controls database provides various project management capabilities. All these data inputs are related to each other via a component number. Since this data is stored in an RDBMS, it is quickly and easily accessed through Structured Query Language (SQL) queries.

\section{CONSTRUCTION CAE}

All data needed by each functional area of the Construction CAE system is stored in an ORACLE (TM) [2] RDBMS. This database is partitioned into five separate areas (see figure 1).

One partition, called the model technical database (MTDB) stores all of the CAD model data for all of the designed components in the model. This data is 
generated in a $C A D$ modeling system and transferred into the database by the Construction CAE preprocessor. This section of the database includes tables for modeled c om ponent s, primitives, and vertices - all the data necessary to accurately represent the model in a graphic display.

The second partitioned area is called the Computer Aided Engineering (CAE) area. This area contains the detail event data needed to produce animated sequences. Events show the state of a component as it moves from a laydown area to its final installation position. The assignment of events to components takes place inside the Simulator portion of the Construction CAE system.

The third area contains the CPM data. CPM data includes the top-down planning hierarchy controlling the start of each component's event chain. This data is output by both the Simulator and an external CPM processor. Top-down planning is discussed in a later section.

A fourth and fifth partitioned areas of the database contain WBS and commodity database (CDB) data. These areas are used in cost tracking and control and are discussed in greater depth in a later section.

\section{The Simulator}

The Simulator portion of the Construction CAE system is an interactive graphic planning tool. The Simulator uses data from the MTDB, CAE, and CPM areas of the database. Data for components in an area of the model specified by the user is queried from the database and written in binary format to temporary data files. The Simulator reads these files upon its invocation.

The Simulator display consists of a combination of top, side, front or back orthographic views and a perspective view of the model in a wireframe or shaded 
rendering mode. The user specifies the rendering mode and up to four views to be displayed concurrently (See figure 2). The display also includes a menu bar across the top of the screen and an information area to the right side of the model display area.

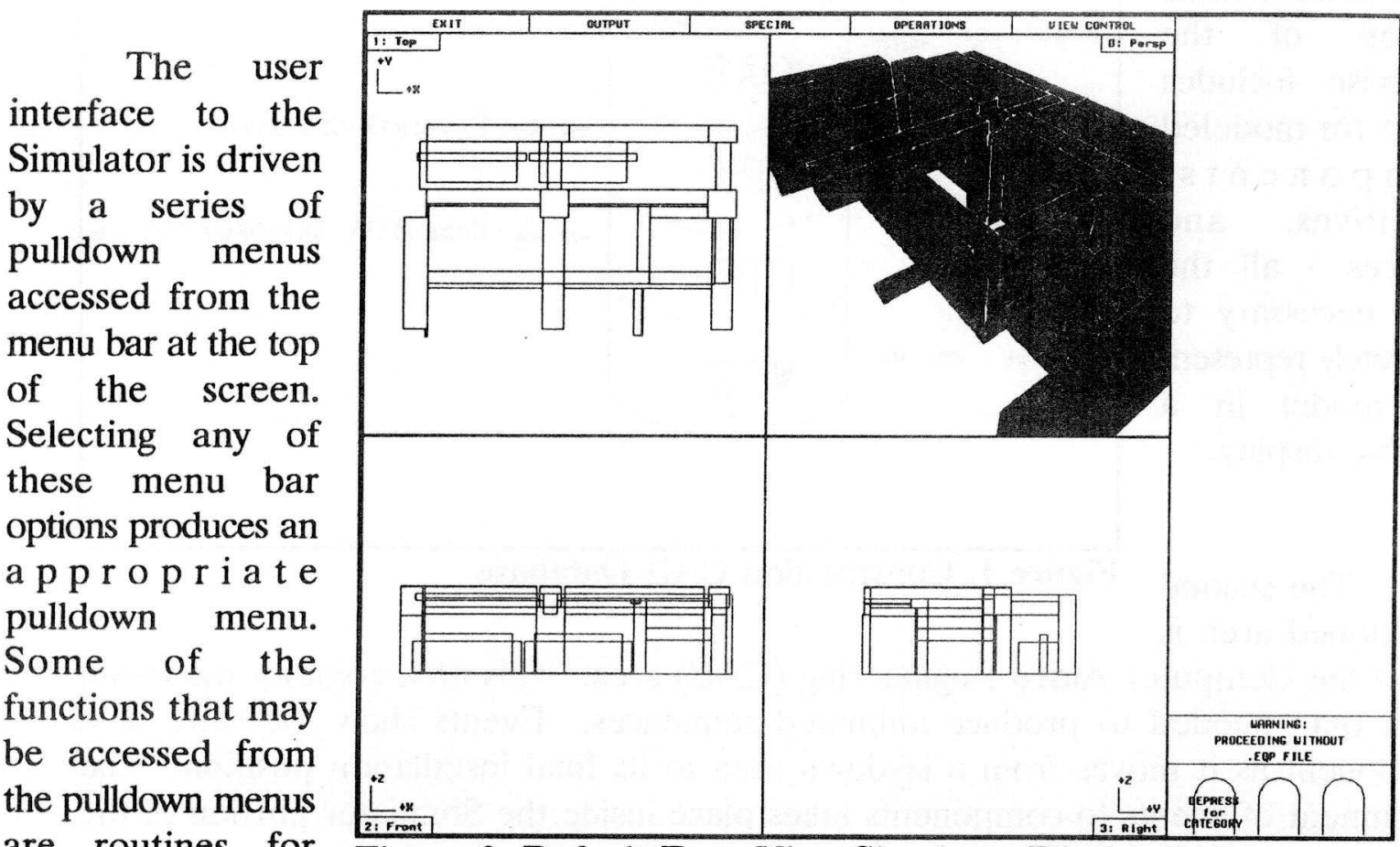

are routines for m e a s u r i n g Figure 2. Default Four View Simulator Display components of the model, toggling the visibility of the components, and making annotations on the screen.

The simplest use of the Simulator is as a visualization tool. By selecting options from the "View Control" pulldown menu, the user may employ the mouse to drag the location of the eye point, or camera, from one position to another in the orthographic views. This creates the sensation of "walking through" the model in the perspective view. By specifying that the perspective view be in shaded rendering mode, the "walk through" can be made very realistic.

The dominant area of Simulator functionality is the "event definitions" feature accessed from the "Operations" pulldown menu. In this area the user defines the chain of events for an item leading up to its installation. The types of events that may be defined include "translate" events to move a component from point to point and "rotate" events to re-orient a component in place. Other event types are "work operation" to allow for the time needed to perform activities on a component to prepare it for installation, "join" events to indicate the time when a component joins with other components to form a module, and "install" events designating the time a model component moves to its final installed location. 
Another group of events allows a component to be picked up, carried, and manipulated in a realistic manner by material handling equipment. Material handling equipment is modeled and entered into the Simulator in a manner paralleling that of the model data. Additional information defining the types and limitations of movement for each component of a type of material handling equipment is also utilized by the Simulator to give it realistic motion.

Relationships between events may be defined by assigning dependencies between events. Dependencies may be start-start (SS), start-finish (SF), finish-finish (FF), or finish-start (FS). A window invoked from an option on the event definitions menu displays a graph of event chains and allows the user to graphically assign these dependencies using the mouse (see figure 3 ).

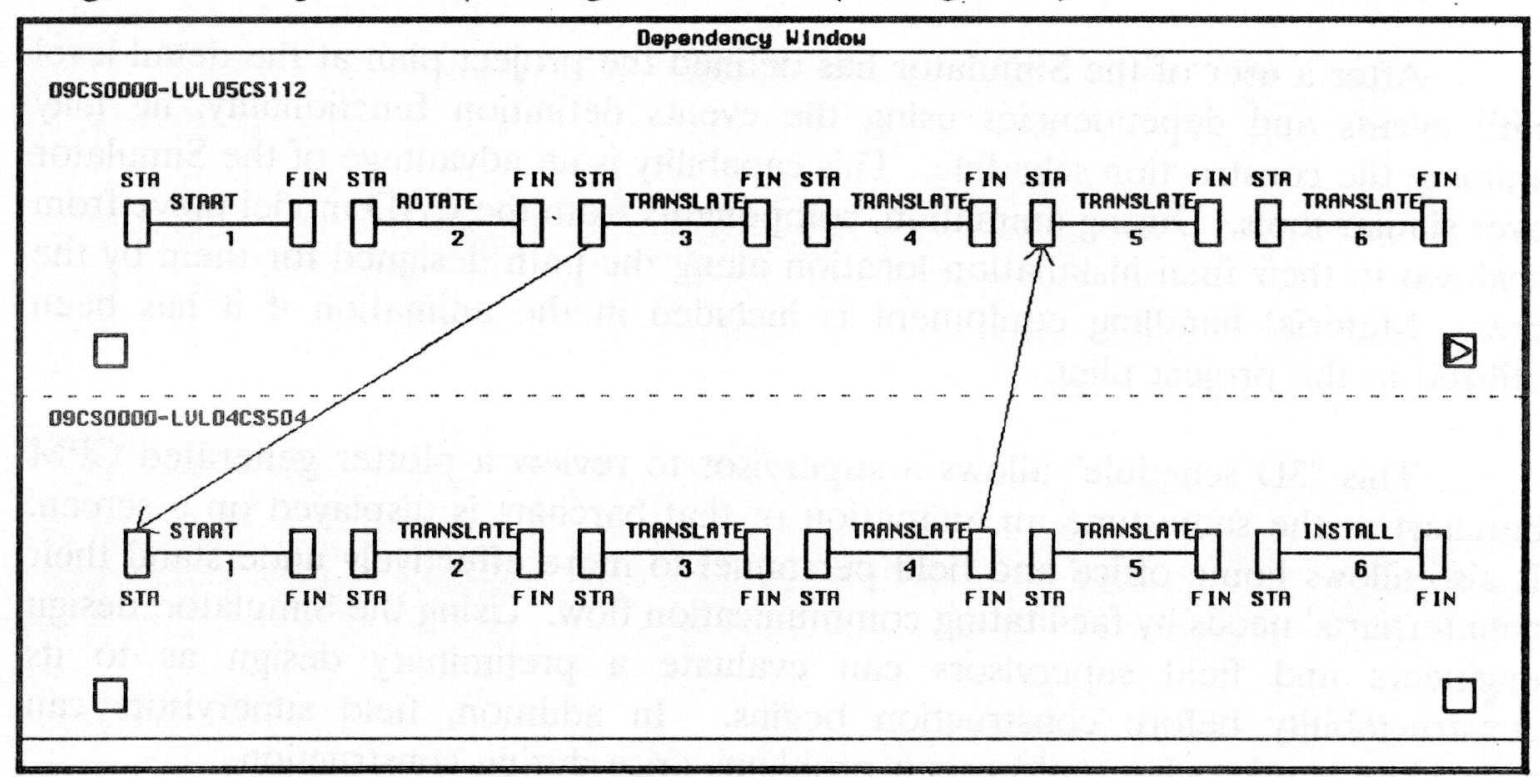

Figure 3. Interactively Assigning Dependencies

\section{Project Control Tools}

Project Planning is accomplished in a top-down manner. Project objectives are the highest level plan and are expanded through successive levels of decreasing abstraction, resulting in detail plans. Events and the dependencies between events are at the greatest level of detail. The possible plan levels are:

milestone Level

-

Intermediate Level 


\section{Detail Level}

Each level is linked by dependency relationships to higher levels in the structure. This allows duration changes at the detail level to be propagated through the hierarchy. Objectives are represented by the milestone level in the plan hierarchy. The milestone plan is the most general and shows when important events are expected to occur during the course of a project. As such, milestone start times are controlled by finish times of activities in any lower level in the plan.

\section{Schedule Animation}

After a user of the Simulator has defined the project plan at the detail level with events and dependencies using the events definition functionality, he may animate the construction schedule. This capability is an advantage of the Simulator over similar tools. During animation, components from the CAD model move from laydown to their final installation location along the path designed for them by the user. Material handling equipment is included in the animation if it has been utilized in the project plan.

This "3D schedule" allows a supervisor to review a plotter generated CPM barchart at the same time an animation of that barchart is displayed on a screen. It also allows home office and field personnel to more effectively understand their counterparts' needs by facilitating communication flow. Using the Simulator, design engineers and field supervisors can evaluate a preliminary design as to its constructability before construction begins. In addition, field supervisors can reevaluate a plan when unforeseen problems arise during construction.

\section{Project Scheduling}

Schedules can be generated by calculating a resource schedule or by simply applying a CPM schedule processor. Both facilities are available as options within Construction CAE.

Schedule data can be extracted from any level in the plan hierarchy and transferred to a commercially available CPM-based scheduling tool for management reporting. It is a goal of Construction CAE to incorporate schedule data into a larger master plan housed on a commercially available schedule processor.

\section{Chronicling and Analysis}

Two commercially available tools have been interfaced with Construction $\mathrm{CAE}$ to assist a cost/schedule engineer in chronicling and analysis. Chronicling 
refers to defining installation status for components in the model while analysis is evaluation of the installation status in search of potential changes in scope and their resulting affects on the current schedule. The tools utilized are:

The Commodity Database (CDB), a project controls database.

- The Project Management System (PMS), a WBS cost control system.

The CDB system is a computerized project control tool for monitoring the design and construction status of project physical scope (commodities), documents, and tasks on medium to large size projects with the capability for varying contract types.

The CDB currently receives engineering designed data directly from Construction CAE. In addition, the CDB transfers construction status and tag weight data to Construction CAE for project status. This cycle of data transfer provides a project with a comprehensive project controls system.

The CDB system is composed of a set of independent modules. This allows a project to select from the system only those options which are necessary to support its operation. The CDB system is designed so that the database system will function as an integrated system within the limits of data available. Some functional capabilities of the $\mathrm{CDB}$ include:

mphysical Scope Control - Tools to identify, quantify and monitor the total physical project scope.

Drawing/Document Control - Tools to identify and monitor the issuance history of all project documents.

- Commodity Statusing - Tools to track the design, procurement and construction installation status of all project commodities.

- Engineering Indices - Tools to relate engineering design information for commodities with agreement, drawing, specifications and calculation data to produce commodity index reports.

-Work Package Control - Tools to relate all components of a construction work package such as commodities, drawings and specs.

Labor/Quantity Control

- Task Tracking 


\section{- System Completion Statusing}

The WBS Cost System utilized within Construction CAE, a commercially available SYNERGY (TM) [3] module called Performance Measurement System (PMS), provides capability to meet governmental reporting requirements according to the Cost and Schedule Control System Criteria (CSCSC). Commodity quantity installation over time is extracted from Construction $\mathrm{CAE}$ and loaded into a work breakdown structure. The budgeted cost of work scheduled (BCWS) over time can be calculated and reported. Actual manhours and dollars, part of the status capabilities of the CDB, can also be loaded. Earned progress can be calculated within the WBS.

\section{CURRENT STATE OF DEVELOPMENT}

Construction CAE has completed initial development (as of January 1989) and is undergoing testing on several projects in the United States and Japan. As a result of this testing, the planning and scheduling portions of the system have been reevaluated. The hierarchical planning mechanism and a resource constraining processor discussed in this paper are a result of that evaluation and will be developed during 1989. Other items discussed: ties to a CAD system; schedule animation; ties to a project controls database; are currently functional.

Although it is still very early in the life of the Construction CAE system, positive feedback has been received by the software designers. Some of this feedback has come from outside the construction industry where other applications of the system may exist. Among these potential applications are plotting paths for autonomous robots, animating manufacturing processes, and planning maintenance procedures during power plant outages.

\section{REFERENCES:}

1. Barrie,D.S. and Paulson,B.C., Professional Construction Management, McGrawHill, Inc., 1984.

2. ORACLE relational database management system, Oracle Corporation, 1986.

3. SYNERGY project control system, Bechtel Power Corporation, 1988. 